\title{
PNGVL3-hICD Vaccine
}

National Cancer Institute

\section{Source}

National Cancer Institute. pNGVL3-hICD Vaccine. NCI Thesaurus. Code C61146.

A plasmid DNA cancer vaccine encoding the intracellular domain (ICD) of the HER-2/neu proto-oncogene. Upon administration and after cellular uptake by skin or muscle cells, the pNGVL3-hICD vaccine plasmid expresses the HER-2/neu protein, which, after intracellular processing, may elicit both antigen-specific cytotoxic T-lymphocyte (CTL) and humoral immune responses ag ainst tumor cells expressing HER-2. The HER-2/neu ICD protein is highly immunogenic and, as a subdominant epitope, may be associated with decreased immune tolerance. 